\title{
Effect of regulated expression of human cyclooxygenase isoforms on eicosanoid and isoeicosanoid production in inflammation
}

\author{
B.F. McAdam, ${ }^{1}$ I.A. Mardini,,${ }^{1,2}$ A. Habib,${ }^{1}$ A. Burke, ${ }^{1}$ J.A. Lawson, ${ }^{1}$ S. Kapoor,${ }^{3}$ \\ and G.A. FitzGerald 1,3 \\ ${ }^{1}$ Center for Experimental Therapeutics, \\ ${ }^{2}$ Department of Anesthesia, and \\ ${ }^{3}$ General Clinical Research Center, University of Pennsylvania, Philadelphia, Pennsylvania, USA
}

Address correspondence to: Garret A. FitzGerald, Center for Experimental Therapeutics, University of Pennsylvania, 811 BRB II/III, 421 Curie Boulevard, Philadelphia, Pennsylvania 19104-6160, USA. Phone: (215) 898-1185; Fax: (215) 573-9135; E-mail: garret@spirit.gcrc.upenn.edu.

B.F. McAdam's present address is: Division of Cardiology, Vanderbilt University Medical Center, Nashville, Tennessee, USA. A. Habib's present address is: Institut Nationale de la Santé et de la Recherche Médicale, Unité 348, Hôpital Lariboisiere, Paris, France.

Received for publication February 3, 2000, and accepted in revised form April 6, 2000.

To examine the role of cyclooxygenase (COX) isozymes in prostaglandin formation and oxidant stress in inflammation, we administered to volunteer subjects placebo or bolus injections of lipopolysaccharide (LPS), which caused a dose-dependent increase in temperature, heart rate, and plasma cortisol. LPS caused also dose-dependent elevations in urinary excretion of 2,3-dinor 6-keto PGF $_{1 \alpha}(\mathrm{PGI}-\mathrm{M})$ and 11-dehydro thromboxane $\mathrm{B}_{2}(\mathrm{Tx}-\mathrm{M})$. Platelet COX-1 inhibition by chronic administration of lowdose aspirin before LPS did not alter the symptomatic and febrile responses to LPS, but the increment in urinary PGI-M and Tx-M were both partially depressed. Pretreatment with ibuprofen, a nonspecific COX inhibitor, attenuated the febrile and systemic response to LPS and inhibited prostanoid biosynthesis. Both celecoxib, a selective COX-2 inhibitor, and ibuprofen attenuated the pyrexial, but not the chronotropic, response to LPS. Experimental endotoxemia caused differential expression of the COX isozymes in monocytes and polymorphonuclear leucocytes ex vivo. LPS also increased urinary $\mathrm{iPF}_{2 \alpha^{-}}$ III, $\mathrm{iPF}_{2 \alpha}$-VI, and 8,12-iso-iPF $2 \alpha-\mathrm{VI}$, isoprostane (iP) indices of lipid peroxidation, and none of the drugs blunted this response. These studies indicate that (a) although COX-2 predominates, both COX isozymes are induced and contribute to the prostaglandin response to LPS in humans; (b) COX activation contributes undetectably to lipid peroxidation induced by LPS; and (c) COX-2, but not COX-1, contributes to the constitutional response to LPS in humans.

J. Clin. Invest. 105:1473-1482 (2000).

\section{Introduction}

Prostaglandins (PGs) are potent, evanescent mediators of both inflammation and thrombosis $(1,2)$. Their formation from arachidonic acid is catalyzed by the enzyme PGG/H synthase, colloquially known as cyclooxygenase (COX; ref. 3). Mammalian cells contain two isoforms of COX (2-4). They are structurally homologous and have similar kinetic properties, but they are differentially regulated $(2,5)$. COX-1 is expressed in almost all tissues, including platelets, and its PG products are thought to mediate physiological responses, such as vascular homeostasis and gastroprotection. COX-2, although often undetectable in resting cells, is readily induced as an immediate early gene in response to cytokines, growth factors, phorbol esters, and bacterial LPS. It is expressed in monocytes, macrophages, neutrophils, and endothelial cells at sites of inflammation $(2,5-10)$. These observations suggest that COX-2 is the source of prostaglandins mediating the inflammatory response, thus affording a rationale for the development of selective inhibitors of this isozyme $(5,6,11)$. Indeed, drugs that preferentially inhibit COX-2 in vitro substantially depress LPS-evoked increases in prostaglandin formation in in vitro systems and in animal models of inflammation in vivo $(12,13)$.

Endotoxin, the LPS outer membrane constituent of Gram-negative bacteria, is well described as a potent stimulus to COX-2 induction, both in vitro and in vivo $(14,15)$. LPS may be administered safely as a bolus to humans under controlled conditions (16-19); however, this experimental model differs from clinical sepsis in important respects, most notably in the transience and magnitude of the endotoxemia. Although the model may help elucidate the role of biochemical pathways in the response to LPS, it does not predict the clinical usefulness of inhibiting those pathways in established, chronic sepsis. Thus, although prior administration of isoform nonselective inhibitors modulate the effects of LPS administration $(20,21)$, a controlled clinical trial of an isoform nonspecific non- 
steroidal anti-inflammatory drug (NSAID) failed to modify clinical outcome in established sepsis (22). Despite prior study of prostanoids in the response to LPS, characterization of the prostanoid response and the relative contribution of the individual isozymes to this response are unknown. To investigate this phenomenon, we utilized chronic administration of lowdose aspirin as a probe for COX-1 inhibition (23), the biochemically selective inhibitor celecoxib as a probe for COX-2 (24), and ibuprofen as a nonselective inhibitor of both isozymes. We measured urinary excretion of thromboxane and prostacyclin metabolites as indices of LPS-induced eicosanoid generation (25).

Additional to its effects on PG formation, experiments in vitro suggest that oxidant signaling pathways may contribute to the effects of LPS. To address the possibility that LPS evokes free radical generation in humans, we have studied its effects on isoprostane (iP) formation. The iPs are free radical catalyzed PG isomers (26). They are formed in situ in cell membranes at the site of free radical attack, are subsequently cleaved by phospholipases, circulate, and are excreted in urine (27-29). Indeed, measurement of these compounds in urine offers a quantitative, time-integrated, noninvasive reflection of lipid peroxidation in vivo $(26,30,31)$. In the present study, we have evaluated the effect of controlled administration of LPS on urinary excretion of three distinct $\mathrm{F}_{2}$-iPs, $\mathrm{iPF}_{2 \alpha}$-III (formerly known as 8iso $\mathrm{PGF}_{2 \alpha}$; ref. 30) $\mathrm{iPF}_{2 \alpha}$-VI (formerly known as $\mathrm{IPF}_{2 \alpha}$-I; ref. 29) and 8,12-iso-iPF ${ }_{2 \alpha}-\mathrm{V} 1$ (32), the most abundant urinary $\mathrm{F}_{2}-\mathrm{iP}(33)$.

\section{Methods}

Subjects. Twenty-six nonsmoking healthy volunteers (mean age: 29.2 years; range: $19-34$ years) were evaluated for inclusion in these studies. Subjects were not permitted to take any drugs for at least 2 weeks before the study, and abstention from use of aspirin or NSAIDs was confirmed by measurement of serum thromboxane $(\mathrm{Tx}) \mathrm{B}_{2}$ drawn on the day immediately before LPS administration. Serum $\mathrm{TxB}_{2}$ was also used to confirm compliance with aspirin and ibuprofen administration. An additional 18 healthy volunteers (11 males) were recruited for evaluation of COX-2 isozyme specific inhibition on response to LPS administration.

Study design. First, we performed a dose-response, placebo-controlled study to characterize the effect of endotoxin administration on the biosynthesis of PGs and the generation of iPs. Nine male and nine female volunteers were randomized under double-blind conditions, to receive a bolus injection of saline or one of two doses ( 2 or $4 \mathrm{ng} / \mathrm{kg}$, respectively) of reference Escherichia coli endotoxin. Purified LPS from E. coli (US Reference E. coli endotoxin lot E.C.-5; Bureau of Biologics, Food and Drug Administration [FDA], Bethesda, Maryland, USA; provided by H.D. Hochstein) with a specific activity of 5 $\mathrm{U} / \mathrm{ng}$, was supplied as a sterile dry powder. This was reconstituted according to FDA prescription just before use. Subjects were admitted to the General Clinical
Research Center (GCRC) the evening before the study, and an intravenous infusion of saline was commenced. The volunteers were fasted overnight until 8 hours after LPS administration. Two hours before administration of the endotoxin, an intravenous catheter was sited from which serial blood samples were drawn. Patency of the catheter was maintained with flushes of saline. The endotoxin was administered as a bolus into the peripheral line at 0800 hours and flushed with $10 \mathrm{~mL}$ of saline. Volunteers were closely monitored and evaluated during the course of the study with frequent measurements of vital signs. The electrocardiogram was continuously monitored. The subjects were observed in the GCRC overnight and were discharged at 24 hours upon completion of the protocol. In a second study, aspirin $(81 \mathrm{mg})$ was administered for 10 days before LPS injection to four volunteers (two males) to ensure maximal, cumulative inhibition of platelet COX-1. This schedule completely inhibited constitutively expressed COX-1 activity in platelets (23). Four volunteers were also administered ibuprofen $(800 \mathrm{mg})$, an isoform nonspecific inhibitor (34), acutely 30 minutes before LPS injection. In a third study, each of 18 volunteers (11 males) was randomized to receive celecoxib (800 $\mathrm{mg}$ ), ibuprofen (800 mg), or placebo before administration of an intermediate dose of LPS ( $3 \mathrm{ng} / \mathrm{kg})$ to explore further the endotoxin dose-response relationship.

Urinary eicosanoid and isoeicosanoids. In all protocols, urine samples were collected before LPS administration and at set intervals (every 2-4 hours) for the following 24 hours. Urinary Tx-M, PGI-M, $\mathrm{PPF}_{2 \alpha}-\mathrm{III}, \mathrm{PPF}_{2 \alpha}-\mathrm{V} 1$, and 8,12-iso-iPF $2 \alpha-V I$ were measured using GC/MS, as described previously $(24,30,33,35)$. Samples for cortisol and serum $\mathrm{TxB}_{2}$ were obtained at baseline and 2, 4, 6,12 , and 24 hours after LPS injection (23).

Expression of COX isozymes ex vivo. Forty milliliters of blood were drawn immediately before and at $30 \mathrm{~min}$ utes, 90 minutes, 3 hours, and 24 hours after LPS injection. Monocytes and neutrophils were separated and harvested for the study of COX expression. All cell culture buffers and media were purchased from GIBCO BRL. Protein A-Sepharose CL-4B was obtained from Pharmacia (Bromma, Sweden). Monoclonal anti- $\beta$ actin, sheep anti-mouse IgG coupled to peroxidase, LPS E. coli 026:B6, and Histopaque were from Sigma Chemical Co. (St. Louis, Missouri, USA). The LAL kit for endotoxin testing was obtained from Pytotell (Woods Hole, Massachusetts, USA).

Blood was drawn into sterile polypropylene syringes containing heparin $(20 \mathrm{U} / \mathrm{mL}$ final concentration) for isolation of neutrophils and monocytes. Monocytes were then isolated by density gradient centrifugation through Histopaque-1077 (Sigma Chemical Co.) for 40 minutes at $1,200 \mathrm{~g}$. The mononuclear fraction was resuspended in PBS and washed three times before its incubation in RPMI 1640 media (GIBCO BRL), 5\% heat-inactivated FBS, together with $2 \mathrm{mM}$ glutamine, $100 \mathrm{U} / \mathrm{mL}$ penicillin, and $100 \mu \mathrm{g} / \mathrm{mL}$ streptomycin at $37^{\circ} \mathrm{C}, 5 \% \mathrm{CO}_{2}$ for $45-60$ minutes. Adherent monocytes were then removed after incubation of the suspension 
cells in a gelatin coated flask. The nonadherent cells were then washed off, and the remaining cell population, which was $95 \%$ pure monocytes, was removed from the plates with a $0.05 \%$ EDTA/PBS solution. Thus, the mean time blood was manipulated ex vivo sampling was 2 hours. Separation procedures were conducted at $37^{\circ} \mathrm{C}$ for $45-60$ minutes, and thereafter, cells were maintained at $4^{\circ} \mathrm{C}$ to minimize activation. Monocytes were identified by morphology and Giemsa staining and were subsequently lysed for 30 minutes in icecold buffer (50 mM Tris/ $\mathrm{HCl}$ [pH 8.0], $1 \mathrm{mM}$ EDTA containing leupeptin, aprotinin, $0.5 \%$ SDS, $10 \%$ NP-40, AEBSF, soybean trypsin inhibitor and $0.05 \%$ DTT), scraped with a rubber policeman and centrifuged $\left(10,000 \mathrm{~g}\right.$ for 15 minutes at $\left.4^{\circ} \mathrm{C}\right)$. The supernatants were stored in liquid nitrogen until analysis by Western blotting for COX-1 and immunoprecipitation for detection of COX-2 (described later here). Protein content was assayed using a microbichoninic assay (Pierce Chemical Co., Rockford, Illinois, USA) with bovine albumin as a standard.

Neutrophils were isolated over 1 hour at $4^{\circ} \mathrm{C}$ in a similar fashion, except that serial differential centrifugation steps were used to purify the harvest of these cells, as described previously here. Although these isolates were relatively (> 95\%) pure, they had some contamination with platelets. Thus, examination for the presence of altered expression of COX-1 was not possible. The pelleted neutrophils were then lysed in Tris/EDTA buffer containing protease inhibitors and stored in liquid nitrogen until analysis. All equipment used for blood collection and cell culture materials was sterilized, and the media solutions were filtered to minimize bacterial contamination. In addition, all materials and labware were tested with the Limulus Ameobocyte Lysate test (Pyrotell) to exclude a contaminant effect of exogenous LPS on the cell suspensions.

COX expression. COX-1 protein was quantitated by Western analysis as described previously (36). Briefly, monocyte lysate $(20 \mu \mathrm{g})$ were mixed with Laemmli reagent (final concentration $2 \%$ SDS wt/vol, $10 \%$ glycerol wt/vol, and $0.005 \%$ pyronin red wt/vol) under reducing conditions ( $4 \% \beta$-mercaptoethanol), heated to $95^{\circ} \mathrm{C}$ for 10 minutes, and subjected to SDS-PAGE using $10 \%$ and $5 \%$ acrylamide for the separating and stacking gels, respectively. Proteins were electroblotted onto nitrocellulose membranes (Schleicher \& Schuell, Gottingen, Germany) with a semidry transfer unit (Hoefer Scientific Instruments, San Francisco, California, USA). Transfer was performed using $25 \mathrm{~mm}$ Tris, $192 \mathrm{mM}$ glycine buffer ( $\mathrm{pH} 8.5$ ) containing $0.01 \%$ SDS, and $20 \%$ methanol for 2 hours at $200 \mathrm{~mA}$. Blots were saturated overnight at $4^{\circ} \mathrm{C}$ with fat-free dry milk in Tris buffered saline (50 mM Tris- $\mathrm{HCl}$ [pH 7.5], $250 \mathrm{~mm}$ $\mathrm{NaCl}$, and $0.1 \%$ Tween 20 ) and incubated with $5 \mu \mathrm{g} / \mathrm{mL}$ of mouse $\mathrm{mAb}$ specific for COX-1 (kindly provided by the late J. Maclouf) for 1 hour at room temperature. An $\mathrm{mAb}$ to $\beta$-actin served as an internal standard for control of protein loading. The blots were then washed six times, each for 10 minutes, in Tris buffer without milk and then further incubated with anti-mouse immunoglobulin conjugated with horseradish peroxidase $(1 / 2,500)$ for 1 hour at room temperature. The excess of the second antibody was washed off six times with Tris buffer. Chemiluminescence substrates were added to reveal positive bands. These were visualized after exposure to Hyperfilm ECL (Amersham Life Sciences Inc., Arlington Heights, Illinois, USA) and quantification was performed by laser densitometry (Pharmacia). The results with the $\mathrm{mAb}$ to $\mathrm{COX}-1$ were confirmed using a rabbit polyclonal antibody raised against the same protein (ram-purified COX). Both antibodies are specific for COX-1 as described previously (37) and confirmed by us in COS-7 cells stably transfected with cDNAs for either human COX-1 (a gift of C. Funk, University of Pennsylvania, Philadelphia, Pennsylvania, USA) or COX-2 (a gift of S. Prescott, University of Utah, Salt Lake City, Utah, USA), comparing them to an $\mathrm{mAb}$ raised to residues $570-595$ of COX-2 (data not shown).

COX-2 protein was examined in neutrophil and monocyte lysates and measured by immunoprecipitation. Briefly, $15 \mathrm{mg}$ of protein A-Sepharose CL-4B was incubated overnight at $4^{\circ} \mathrm{C}$ with $50 \mu \mathrm{L}$ of specific polyclonal anti-COX-2 peptide antibody (again, kindly provided by J. Maclouf) in $500 \mu \mathrm{L}$ of buffer $(50 \mathrm{mM}$ Tris$\mathrm{HCl}$ [pH 8], $150 \mathrm{mM} \mathrm{NaCl}, 1 \% \mathrm{NP}-40,0.5 \%$ deoxycholic acid, and $0.1 \%$ SDS). After washing five times with this buffer, protein A-Sepharose, preincubated with the antibody, was then incubated with the cell lysates $(100 \mu \mathrm{g})$ for 2 hours at $4^{\circ} \mathrm{C}$. After pelleting the supernatant, the protein A-Sepharose antibody mixture was added and reincubated for 3 hours at $4^{\circ} \mathrm{C}$. The supernatant-protein A-Sepharose was then washed five times with the same buffer, mixed with Laemmli reagent under nonreducing conditions, heated for 10 minutes at $90^{\circ} \mathrm{C}$, subjected to SDS-PAGE, and transferred to nitrocellulose membranes. Membranes were then subjected to Western blot analysis using $5 \mu \mathrm{g} / \mathrm{mL}$ of anti COX- 2 antibody and secondary anti-mouse antibody $(1 / 5,000)$. Again, this COX antibody is highly isoform specific (38).

Statistical analysis. All results are expressed as mean SEM. The data were subjected to two-way ANOVA as appropriate. Statistical comparisons were made for differences between treatments with LPS or placebo and then compared with the effects of COX inhibitors. Grouped data were initially analyzed for covariance of slopes before calculation of pooled correlation coefficients.

\section{Results}

Systemic and clinical responses. All 12 subjects in the LPS dose-response study who received endotoxin developed fever, headache, malaise, myalgia, nausea, and fatigue. This "flulike" syndrome was induced within 2-3 hours after administration of LPS and had disappeared roughly 10 hours later. The study was otherwise well tolerated by the volunteers. The six subjects who received placebo did not report any symptoms during the corresponding 
period. LPS caused a dose-dependent increase in body temperature. The mean maximal response was observed at 4 hours, reaching $37.6 \pm 0.09^{\circ} \mathrm{C}$ in the low-dose group and $38.3 \pm 0.13^{\circ} \mathrm{C}$ in the subjects who received $4 \mathrm{ng} / \mathrm{kg}$ LPS. The temperatures returned to normal 10 hours after LPS. Only the volunteers who were administered the higher dose of LPS (4 ng/ $\mathrm{kg}$ ) experienced rigors. A dosedependent cardiovascular response was also observed,

a

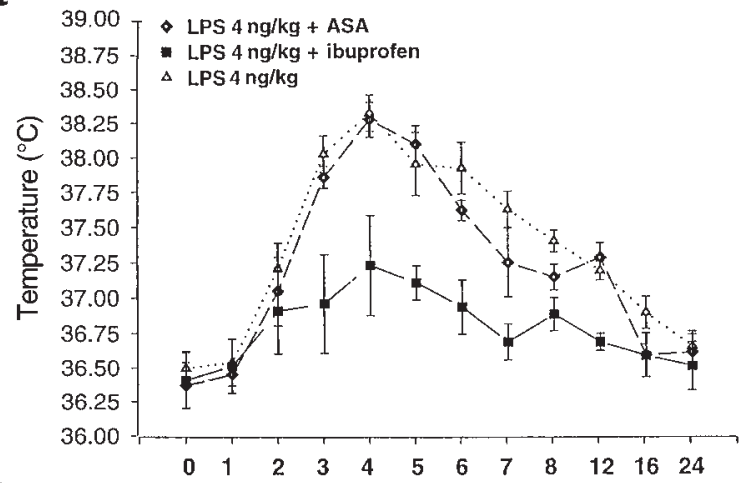

b

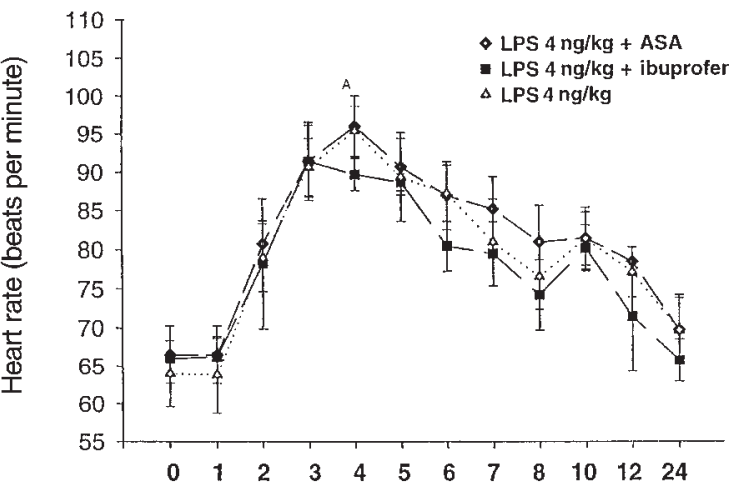

C

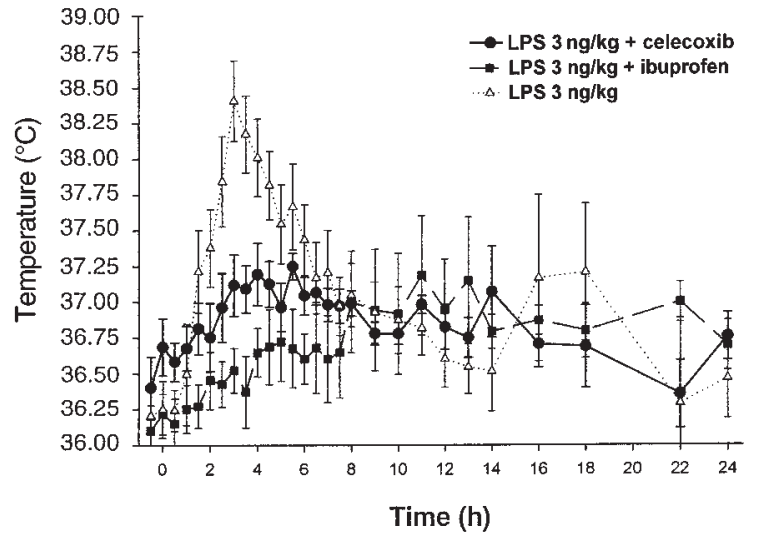

Figure 1

(a) The effect of chronic administration of low-dose aspirin (ASA; 81 $\mathrm{mg}$ for 10 days) and acute administration of the nonselective NSAID ibuprofen ( $800 \mathrm{mg}$ ) on the pyrexial response to $4 \mathrm{ng} / \mathrm{kg}$ LPS. Ibuprofen, but not aspirin, significantly attenuated the pyrexial response to endotoxin $(P<0.01)$. (b) The effect of LPS on heart rate is not altered by either aspirin or ibuprofen ( $\left.{ }^{A} P=N S\right)$. (c) Pretreatment with either celecoxib (800 mg) or ibuprofen $(800 \mathrm{mg})$ significantly $(P<$ 0.01 ) depressed the pyrexial response to LPS ( $4 \mathrm{ng} / \mathrm{kg})$. Body temperature did not differ significantly between subjects receiving celecoxib or ibuprofen before LPS. NS, not significant. with significant increases in heart rate. Blood pressure was unchanged (data not shown). LPS induced a rise in plasma cortisol, when compared with the normal diurnal variation observed in the volunteers who received placebo.

Low-dose aspirin failed to alter the hemodynamic, pyrexial or cortisol responses to LPS (Figures 1, a and b, Table 1). By contrast, none of the volunteers treated with ibuprofen experienced rigors, and only two reported mild headache. The maximal pyrexial response to LPS observed at 4 hours was also reduced by administration of ibuprofen to $37.24 \pm 0.33^{\circ} \mathrm{C}(P<0.05$; Figure 1a) and the cortisol response was attenuated (Table 1). However, ibuprofen failed to alter significantly the heart rate response to LPS (Figure 1b). Similarly, celecoxib significantly attenuated the pyrexial response to LPS (3 $\mathrm{ng} / \mathrm{kg}$ ) from a peak of $38.4 \pm 0.03^{\circ} \mathrm{C}$ in the placebo group to $37.2 \pm 0.02^{\circ} \mathrm{C}$ (Figure 1c), without influencing heart rate. Body temperature was not significantly different in subjects pretreated with celecoxib compared with those pretreated with ibuprofen prior to LPS.

Urinary prostaglandin metabolites. LPS caused a dosedependent increase in the excretion Tx-M. This was evident immediately at 0 to 3 hours after injection and peaked in the 3- to 6-hour collection interval after LPS (Figure 2a). The response to $4 \mathrm{ng} / \mathrm{kg}$ represents an almost fivefold increase over baseline, from $450 \pm 65$ to $2,152 \pm 346 \mathrm{pg} / \mathrm{mg}$ creatinine $(P<0.001)$. There was no change in the excretion of this metabolite in subjects who were given placebo.

When low-dose aspirin was administered before LPS, baseline excretion of the Tx-M was reduced from $425 \pm$ 105 to $150 \pm 32 \mathrm{pg} / \mathrm{mg}$ creatinine $(P<0.05)$. Serum thromboxane $\mathrm{B}_{2}$, an index of the capacity of platelet COX-1, was inhibited by more than $95 \%$ compared with pretreatment levels and remained so for the rest of the study (Table 2). Aspirin also markedly reduced the peak LPS-stimulated increment in excretion of Tx-M (1,705 \pm 310 vs. $172 \pm 29$ $\mathrm{pg} / \mathrm{mg}$ creatinine; $P<0.001) 0-3$ hours after injection. Despite incomplete suppression of Tx-M (Figure 3a), there was still an almost fourfold increase in Tx-M excretion compared with baseline at 3-6 hours after injection to 645 $\pm 92 \mathrm{pg} / \mathrm{mg}$ creatinine $(P<0.01)$. Pretreatment with ibuprofen, by contrast, both reduced serum $\mathrm{TxB}_{2}$ by $95 \%$ and completely inhibited urinary Tx-M (Figure 3a). Thus, COX-2 appears to contribute to a discernible extent to the increase in Tx biosynthesis evoked by LPS. Indeed, ibuprofen depressed urinary Tx-M 3-6 hours after LPS to below that observed after pretreatment with aspirin (Figure 3a). Urinary Tx-M had returned to baseline by 24 hours after LPS, reflecting the time course of recovery of serum $\mathrm{TxB}_{2}$. This is consistent with reversible inhibition of both COX isoforms by ibuprofen.

To address the potential source of LPS-stimulated Tx biosynthesis, we studied platelet aggregation in vitro (24). Addition of $250 \mathrm{ng}$ arachidonic acid induced platelet aggregation to $79 \pm 4 \%(n=3)$ of maximum. LPS added to platelets had no effect. At a subthreshold concentration $(220 \mu \mathrm{g})$ of arachidonic acid, no response was evident. Addition of $1 \mathrm{ng}, 50 \mathrm{ng}, 500 \mathrm{ng}$, and $10 \mu \mathrm{g}$ of 
LPS to the subthreshold concentration of arachidonic acid failed to evoke an aggregation response different from control in separate experiments using platelets from three distinct volunteers. Similar results were observed with thrombin as a platelet agonist.

LPS also stimulated a dose-dependent increase in urinary PGI-M (Figure 2b). For example, a tenfold increment in PGI-M excretion occurred after administration of LPS ( $4 \mathrm{ng} / \mathrm{kg}$ ). Thus, excretion of PGI-M increased from a baseline of $90 \pm 17$ to $1,375 \pm 287 \mathrm{pg} / \mathrm{mg}$ creatinine. Although urinary PGI-M excretion at baseline tended to be lower after chronic low-dose aspirin (59 \pm $8 \mathrm{pg} / \mathrm{mg}$ creatinine) compared with baseline in the group destined to receive ibuprofen $(117 \pm 28 \mathrm{pg} / \mathrm{mg}$ creatinine) or placebo $(93.7 \pm 18.5 \mathrm{pg} / \mathrm{mg}$ creatinine $)$, these differences were not statistically different $(\mathrm{F}=1.7$; $P>0.1)$. Low-dose aspirin partially inhibited the response of PGI-M to LPS. Ibuprofen, similar to the results with Tx-M, completely inhibited the increase in PGI-M evoked by LPS (Figure 3, b and c). In the third study, LPS at $3 \mathrm{ng} / \mathrm{kg}$ dramatically increased PGI-M excretion with peak concentrations of PGI-M reaching $881 \pm 193 \mathrm{pg} / \mathrm{mg}$ creatinine. By contrast, celecoxib reduced the peak PGI-M response to $55 \pm 21 \mathrm{pg} / \mathrm{mg}$ creatinine. However, this still remained significantly $(P<$ $0.05)$ higher than the peak PGI-M levels $(16 \pm 0 \mathrm{pg} / \mathrm{mg}$ creatinine) attained after pretreatment with ibuprofen (Figure 3c), suggesting a contribution from COX-1.

Urinary isoprostanes. LPS caused a dose-independent increase in excretion of all three iPs, in contrast to its dosedependent effect on urinary prostaglandin metabolite excretion. LPS (4 ng/ kg) caused urinary $\mathrm{iPF}_{2 \alpha}$-III to rise roughly threefold over baseline, from 16.53 to 52.38 $\mathrm{pg} / \mathrm{mg}$ creatinine $(P<0.005)$. The time course and magnitude of this effect were similar in response to the two doses of LPS, and the peak response coincided with the maximal change in body temperature. There was no change in the excretion of $\mathrm{PPF}_{2 \alpha}$-III in those subjects who were given placebo. The more abundant $\mathrm{iPF}_{2 \alpha}-\mathrm{VI}$ also increased roughly threefold, from 71.59 to $1,856.25$ $\mathrm{pg} / \mathrm{mg}$ creatinine after LPS ( $4 \mathrm{ng} / \mathrm{kg} ; P<0.001)$. Urinary iPs returned close to baseline by 12 hours coincident with resolution of the clinical response to LPS (23). Excretion of both urinary iPs was highly correlated $(r=0.69 ; P<$ $0.001)$. This observation is consistent with a common free radical catalyzed route of their generation. In contrast to

Table 1

The time course and effect of LPS (4 $\mathrm{ng} / \mathrm{kg}$ ) or placebo on plasma cortisol $(\mu \mathrm{g} / \mathrm{dL})$ alone and with low-dose aspirin or ibuprofen

\begin{tabular}{lccccc}
\hline Time & $0 \mathrm{~h}$ & $2 \mathrm{~h}$ & $4 \mathrm{~h}$ & $12 \mathrm{~h}$ & $24 \mathrm{~h}$ \\
Placebo & $18.9 \pm 4.2$ & $14.1 \pm 2.9$ & $9.2 \pm 1.9$ & $4.3 \pm 2.1$ & $18.5 \pm 5.3$ \\
LPS $(4 \mathrm{ng} / \mathrm{kg})$ & $19.7 \pm 3.9$ & $21.8 \pm 2.1$ & $32.5 \pm 2.9$ & $4.8 \pm 1.7$ & $16.5 \pm 4.9$ \\
LPS + aspirin & $16.6 \pm 4.3$ & $22.1 \pm 5.1$ & $37.2 \pm 6$ & $5.5 \pm 3.0$ & $16.2 \pm 4.1$ \\
LPS + ibuprofen & $19.4 \pm 5.1$ & $16.6 \pm 4.2$ & $22.9 \pm 4.8$ & $3.8 \pm 1.6$ & $20.3 \pm 6$ \\
\hline
\end{tabular}

Each value represents mean and SEM. Maximal rise in plasma cortisol was observed at 4 hours after LPS injection $(P<0.01)$. Aspirin did not alter this stress response to LPS, whereas ibuprofen caused a modest reduction in peak cortisol levels at 4 hours $(P<0.5)$.

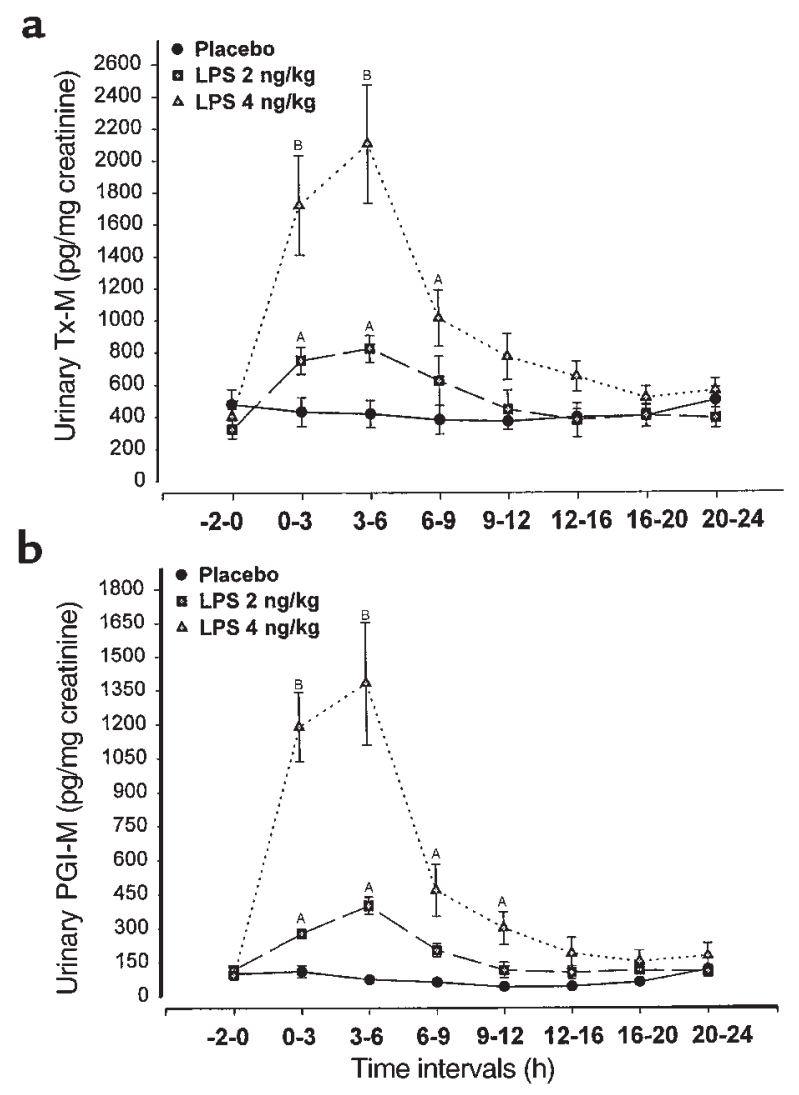

Figure 2

(a) LPS induces an increase in excretion of Tx-M $(P<0.001)$. A doseresponse effect is evident with peak excretion at 3-6 hours. No change in Tx-M excretion was seen with placebo. (b) LPS causes a dose-dependent increase in excretion of PGI-M. The maximal effect at 3-6 hours coincided with the maximal constitutional effects of the endotoxin $(P<0.001)$. ${ }^{A} P<0.05$; ${ }^{\mathrm{B}} P<0.001$.

their effects on LPS-induced PG formation, neither pretreatment with low-dose aspirin nor with ibuprofen significantly depressed the LPS-induced increment in urinary iPF $_{2 \alpha}-\mathrm{III}$ excretion (Figure 4a). Similarly, ANOVA indicated that the increase in urinary 8,12 -iso $\mathrm{iPF}_{2 \alpha}$-VI was not altered by pretreatment with either celecoxib or ibuprofen (Figure 4b).

COX expression. Expression of COX-1 was increased in monocytes ex vivo, most markedly in cells isolated from blood drawn 30 minutes after LPS injection (Figure 5a). The nitrocellulose membrane was stripped and incubated with $10 \mu \mathrm{g} / \mathrm{mL}$ of the purified polyclonal COX-1 and followed by incubation with a donkey anti-rabbit IgG coupled to horseradish peroxidase. Again, time-dependent expression of COX-1 was detected in the monocytes. Immunoprecipitation of COX-2 was not altered in monocytes (Figure $5 \mathrm{~b}$ ). By contrast, COX-2 was readily detected ex vivo in neutrophils at both 90 and 180 minutes after injection (Figure 5c). The ability to detect possible changes in COX-1 in neutrophils was confounded by platelet COX-1 contamination. There was no change in expression of either COX isoform in either cell type after injection of placebo. 


\section{Discussion}

Bacterial LPS induces cells, such as monocytes and macrophages, to elaborate an array of proinflammatory mediators in vitro (39). Induction of ELAM expression and release of the cytokines, TNF- $\alpha$ and IL-8, are dependent on LPS binding protein (40) and enhanced by CD14 (41), whereas LPS activates a Toll-like trans-

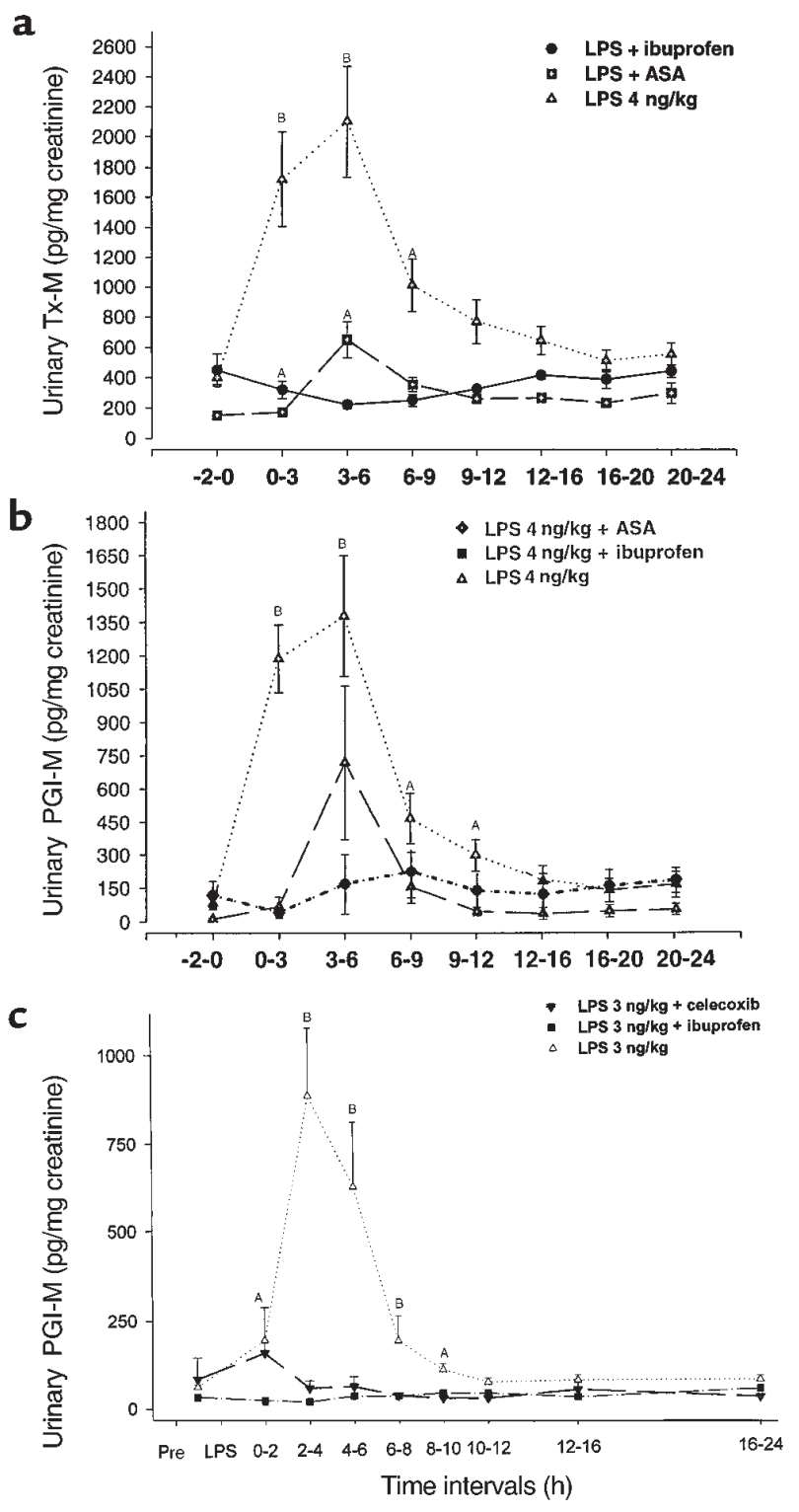

Figure 3

(a) The effect of LPS on Tx-M excretion with selective COX-1 and nonselective COX inhibition. Inhibition of platelet COX-1 with lowdose aspirin partially inhibits excretion of TX-M evoked by $4 \mathrm{ng} / \mathrm{kg}$ LPS. By contrast, ibuprofen completely inhibits the increase in thromboxane biosynthesis induced by the endotoxin $(P<0.01)$. (b) The effect of LPS on PGI-M excretion after selective and nonselective COX inhibition. The increment in PGI-M excretion evoked by $4 \mathrm{ng} / \mathrm{kg}$ LPS is partially inhibited by low-dose aspirin. Nonselective inhibition of both COX isoforms completely inhibits PGI-M excretion. (c) The effect of LPS on PGI-M excretion after selective and nonselective COX inhibition. Although celecoxib substantially depressed the increment in $\mathrm{PGI}-\mathrm{M}$, it is depressed further by ibuprofen. ${ }^{\mathrm{A}} P<0.05$; ${ }^{\mathrm{B}} P<0.001$. membrane receptor, resulting in signal transduction $(42,43)$. Addition of LPS to monocyte/macrophages in vitro results in COX-2 induction and generation of PGs $(14,44,45)$. Surprisingly, the effects of LPS on biosynthesis of PGs in humans is poorly characterized (46).

In the present study, we wanted to address the hypothesis that both COX isoforms contribute to the prostanoid response to LPS in humans. An assumption that is implicit to the development of selective inhibitors of COX-2 is that this isoform accounts for PG formation in human inflammation. The induction of COX-2, but not of COX-1, by inflammatory cytokines in monocyte/macrophages in vitro and the efficacy of COX-2 inhibitors in animal models of inflammation would appear consistent with this hypothesis. Moreover, selective COX-2 inhibitors, such as celecoxib and rofecoxib, are apparently as efficacious as traditional, isoform nonspecific NSAIDs in clinical trials of osteoarthritis and rheumatoid arthritis $(47,48)$. Despite these observations, it remains possible that COX-1 may participate in some human syndromes of inflammation. Hitherto, clinical trials of COX-2 inhibitors and traditional NSAIDs were sized to detect equivalence, not superiority, of either. Furthermore, mice deficient in COX-1, but not COX-2, exhibit a reduction in the inflammatory response to the dermal application of arachidonic acid $(49,50)$, raising the possibility that COX-1 may contribute to inflammation.

In the present studies, we compared chronic treatment with a low-dose $(81 \mathrm{mg})$ of aspirin, a probe for COX-1, with acute administration of COX-2 specific inhibitor, celecoxib, and the nonspecific inhibitor, ibuprofen, on eicosanoid generation. We investigated the effects of LPS and the inhibitors on prostanoid formation by analyzing urinary excretion of major metabolites of $\mathrm{PGI}_{2}$ and $\mathrm{TxA}_{2}$ (23). Although highly selective inhibitors of COX-1 are unavailable for study in humans, chronic administration of low-dose aspirin cumulatively inhibits COX-1-dependent $\mathrm{TxA}_{2}$ formation by platelets (51). It is possible that low-dose aspirin may also have had a partial inhibitory effect on COX-2. Thus, although PGI-M excretion did not differ significantly between the groups before the administration of LPS, it tended to be lower in the aspirin-treated group. However, given this caveat, inhibition of both COX isoforms with ibuprofen or COX-2 inhibition with celecoxib abolished the flulike syndrome and pyrexial response to LPS, whereas low-dose aspirin did not affect these parameters. These observations would be consistent with a dominant role for induced COX-2 in mediating the constitutional response to LPS. Experiments in knockout mice suggest that $\mathrm{PGE}_{2}$, acting via the EP3 receptor, mediates the pyrexial response to LPS (52), and $\mathrm{PGI}_{2}$ also appears important in mediating the pain and swelling evoked by inflammatory stimuli (53). However, although infused, exogenous $\mathrm{PGI}_{2}$ has a chronotropic effect in humans (54), the heart rate response to LPS was unaffected by either low-dose aspirin, celecoxib, or ibuprofen in these experiments. 
LPS administration caused a marked increase in urinary excretion of both Tx-M and PGI-M. Although platelets appear to account for roughly $70 \%$ of urinary Tx-M under physiological condition in humans (23), the contributions from other cell types, most notably monocyte/macrophages $(2,5)$, is likely to increase in inflammation. We found that LPS failed to activate platelets directly and failed to augment the response to subthreshold concentrations of arachidonic acid or thrombin in vitro. Salat et al. (55) also found that LPS failed to activate platelets directly and that the aggregation response to arachidonic acid and collagen in vitro was unaltered. However, they did observe a modest augmentation of the response of platelets to adenosine diphosphate (ADP) by LPS (55). Thus, it is possible that platelets may have contributed to the augmented biosynthesis of TxA $\mathrm{A}_{2}$ induced by LPS.

Given the ready induction of COX-2 by LPS in monocytes observed both by ourselves and others in vitro (45, $56)$, we sought its expression in these cells ex vivo. However, despite harvesting cells at multiple time points, we failed to immunoprecipitate COX-2, using a highly specific antibody (57). This discrepancy may reflect differing concentrations of LPS utilized to induce COX-2 in vitro and in vivo. Others have reported that LPS concentrations in the range of $100 \mathrm{pg} / \mathrm{mL}$ to $10 \mathrm{ng} / \mathrm{mL}$ evoke COX-2 expression in vitro $(14,44)$. We did not perform estimations of circulating LPS attained in the present study; however, levels in the order of $400 \mathrm{pg} / \mathrm{mL}$ have been estimated in patients with clinical endotoxemia (58). These are likely to exceed the concentrations attained in experimental, acute endotoxemia. Interestingly, the kinetics of induction of COX-2 expression that we observed ex vivo in neutrophils were much faster than those observed in vitro. Thus, it is possible that we failed to detect monocyte COX-2 expression ex vivo in the present experiments because we failed to attain and/or sustain LPS concentrations commensurate with those used in vitro. An alternative explanation relates to compartmentalization of the response to LPS (59). Thus, LPS induces a redistribution of leucocytes in the circulating compartment from which blood samples are drawn. Others have described an initial neutropenia followed by a neutrophilia $2.5-3$ hours after LPS injection. This is followed by a prolonged monocytopenia, lasting 6-8 hours (60). We have made similar observations in the present study (data not shown). Studies in vitro have inferred differential sensitivity of COX-2 to induction by LPS in subpopulations of monocytes (61). Thus, it is possible that we failed to detect COX-2 in circulating monocytes ex vivo in the present study because we were sampling circulating populations of monocytes that may differ from the harvested monocytes or monocytoid cells studied previously in vitro. Irrespective of the reason for the discrepancy, the combination of immunoprecipitation and Western blotting afforded particular sensitivity, as well as specificity, to the approach. This is consistent with the marked positivity detected in the positive control experiments performed in vitro and our a
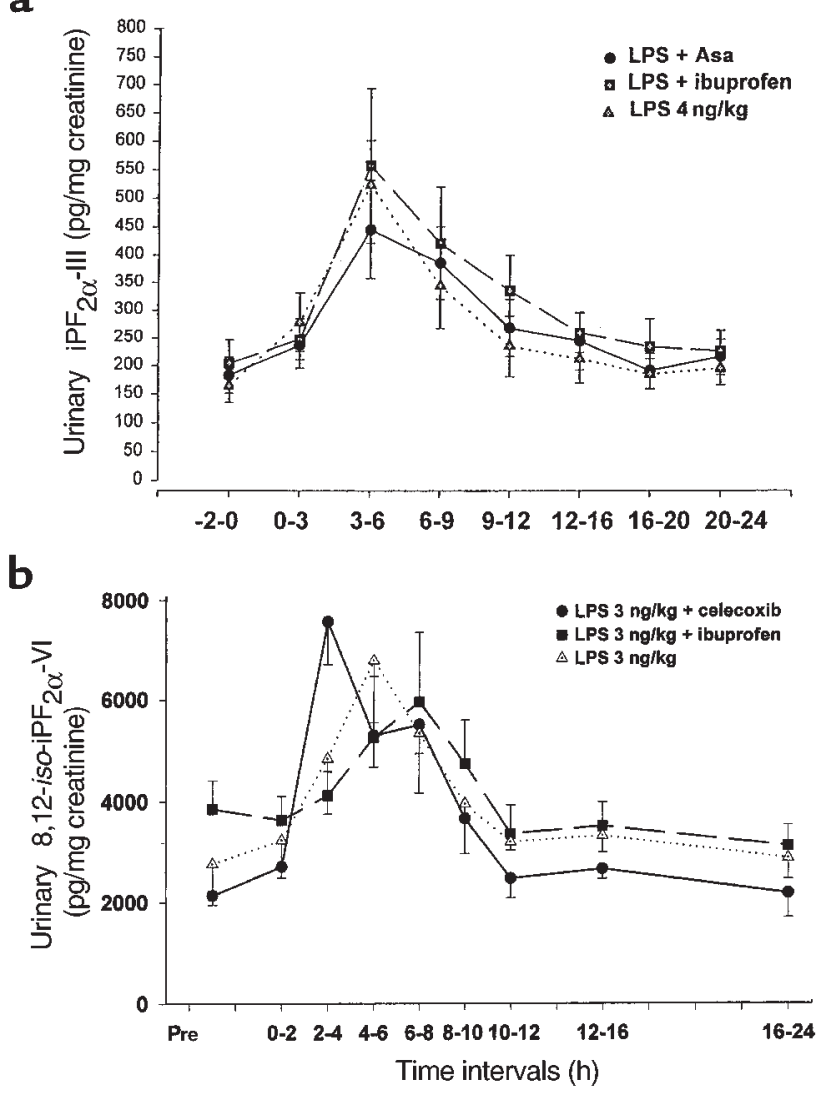

Figure 4

(a) Low-dose aspirin and ibuprofen fail to depress LPS-evoked urinary $\mathrm{PPF}_{2 \alpha}$-III excretion. (b) Celecoxib or ibuprofen fail to depress LPS-evoked urinary 8,12-iso-iPF $2 \alpha-\mathrm{VI}$ excretion. $(P=\mathrm{NS})$.

ability to detect COX-2 in circulating neutrophils. By contrast, using two distinct and highly specific antibodies $(37,38)$, we readily detected time-dependent expression of COX-1 in monocytes ex vivo after LPS administration. These observations raised the possibility that COX-1 also contributes to the prostanoid response to LPS in humans. Although COX-1 may be developmentally regulated (62) and is coexpressed with COX-2 in both atherosclerotic plaque and the synovia of inflamed joints $(63,64)$, its role in these inflammatory syndromes is poorly understood. Clearly, if products of this isozyme

Table 2

The effect of LPS ( $4 \mathrm{ng} / \mathrm{kg}$ ) or placebo on serum $T x B_{2}(\mathrm{ng} / \mathrm{mL})$ alone and with ibuprofen or low-dose aspirin

\begin{tabular}{lcccccc}
\hline & & & & & & \\
Time & Day-7 & $0 \mathrm{~h}$ & $2 \mathrm{~h}$ & $4 \mathrm{~h}$ & $6 \mathrm{~h}$ & $24 \mathrm{~h}$ \\
Placebo & $237 \pm 33$ & $265 \pm 21$ & $277 \pm 25$ & $259 \pm 23$ & $296 \pm 22$ & $260 \pm 31$ \\
LPS (4 ng/kg) & $286 \pm 32$ & $315 \pm 35$ & $294 \pm 22$ & $305 \pm 47$ & $266 \pm 21$ & $229 \pm 40$ \\
LPS+ aspirin & $273 \pm 29$ & $8 \pm 4$ & $10 \pm 6$ & $6 \pm 3$ & $11.5 \pm 9$ & $9.5 \pm 11$ \\
LPS+ ibuprofen $305 \pm 34$ & $269 \pm 23$ & $22 \pm 5$ & $10 \pm 8$ & $17 \pm 13$ & $197 \pm 21$
\end{tabular}

Values are mean \pm SEM. LPS did not increase the capacity of whole blood to generate $\mathrm{TxB}_{2}$ despite significant alterations in the biosynthesis of thromboxane $A_{2}$. Low-dose aspirin for 10 days resulted in $97 \%$ inhibition of serum $T x B_{2}$ before injection of LPS, and no change in this inhibitory effect was observed throughout the study period despite a fourfold increase in its biosynthesis at 3-6 hours after LPS administration. By contrast, ibuprofen caused a similar but reversible inhibitory effect on serum $\mathrm{Tx}_{2}$. 


\section{Figure 5}

(a) Western blot of monocyte COX-1. LPS increases the expression of COX-1 in peripheral blood monocytes. This is maximal in cells that have been isolated from blood drawn at 30 minutes after LPS injection $(4 \mathrm{ng} / \mathrm{kg}$ ). The time from blood withdrawal to detection lasted 2-2.5 hours (see the text). All samples are drawn from a single individual. This result is representative of three separate experiments. (b) Immunoprecipitation of monocyte COX-2. LPS does not alter the expression of COX- 2 in monocytes ex vivo in blood drawn at the time points shown or in monocytes harvested at 1.5, 3, 4, 6, and 12 hours after LPS administration (data not shown). As before, the time for blood withdrawal to detection lasted 2-2.5 hours (see the text). All samples were drawn from a single individual administered $4 \mathrm{ng} / \mathrm{kg}$ LPS, and this study is representative of three separate experiments. $-\mathrm{C}$ denotes a negative control, unstimulated monocytes in vitro. $+\mathrm{C}$ is a positive control and denotes monocytes stimulated with LPS (10 $\mu \mathrm{g} / \mathrm{mL}$ ) in vitro. (c) Immunoprecipitation of neutrophil COX-2. Increased expression of COX-2 was observed in peripheral blood neutrophils isolated from blood drawn at 90 and 180 minutes after LPS injection $(4 \mathrm{ng} / \mathrm{kg}$ ). Time from harvesting to detection was 1 hour (see the text). No alteration in expression was observed after placebo administration. All samples were drawn from a single individual. This result is representative of three separate experiments. a

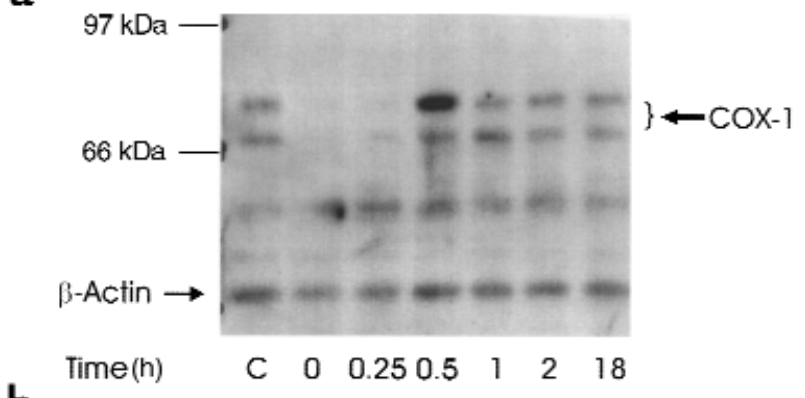

b

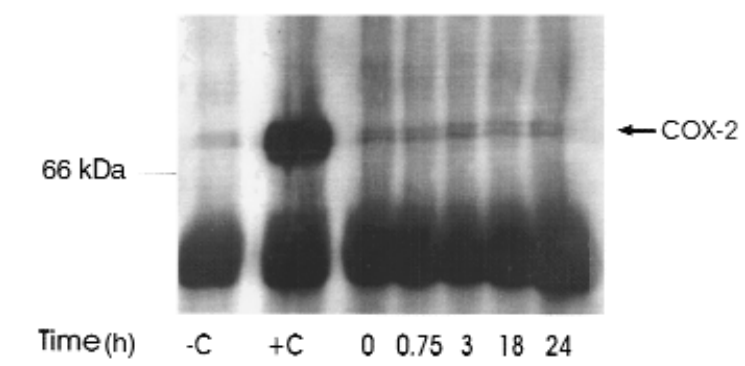

C

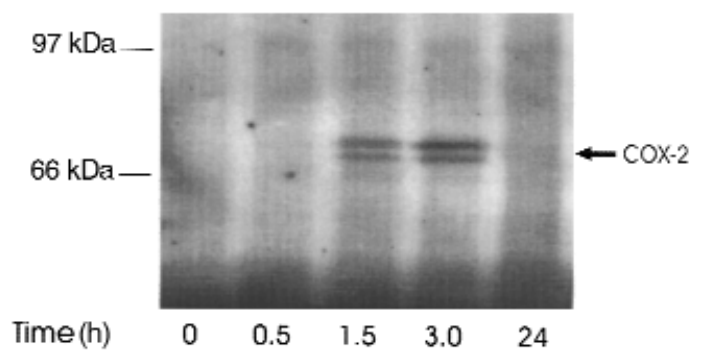

were important contributors to the inflammatory response, it would suggest that isozyme nonspecific inhibition might be desirable in attaining maximum effica$c y$, if unconditioned by consideration of adverse effects. We do not establish a contribution of COX-1-dependent PGs to the constitutional response to experimental endotoxemia. Consistent with this hypothesis, low-dose aspirin partially, but not completely, abolished the Tx-M response to LPS. Maloney and colleagues recently reported the induction of COX-2 expression by LPS in both monocytes and polymorphonuclear leucocytes in vitro (65). In the present study, we observed expression of COX-2 in neutrophils ex vivo after LPS administration. The contribution of COX-2-dependent PG formation to the constitutional response to LPS emphasizes the potential importance of neutrophils as a source of PGs in human inflammation. Although they were more than $95 \%$ pure, these preparations did contain some platelets, precluding us from seeking expression of COX1. However, irrespective of their relative contributions to LPS-evoked prostanoid generation, we document the regulated expression of both COX isoforms ex vivo by an inflammatory stimulus in humans.

Vascular tissue is an important source of $\mathrm{PGI}_{2}$ and urinary metabolite excretion increases on stimulation of the vasculature in humans (66-70). Although COX-1, but not COX-2, is constitutively expressed in endothelial cells in vitro, COX-2 expression is induced by LPS, inflamma- tory cytokines, and growth factors $(71,72)$. Physiological rates of shear also upregulate COX-2 in endothelial cells (73), and structurally distinct, selective COX-2 inhibitors reduce PGI-M excretion to the same extent as do classic NSAIDs in healthy volunteers $(24,34)$. These results are consistent with shear-induced upregulation of COX-2 in endothelial cells largely accounting for urinary excretion of this metabolite under physiological conditions in humans. LPS administration evoked a marked increase in PGI-M excretion in volunteers. This was partially suppressed by administration of low-dose aspirin, implicating COX-1, but completely abolished by ibuprofen. Although pretreatment with celecoxib substantially depresses the PGI-M response to LPS, this effect was incomplete, as indicated by comparison with the suppression achieved by pretreatment with ibuprofen. Thus, similar to the results with Tx-M, both isoforms appear to contribute to the formation of this $\mathrm{PG}$ also in response to LPS, whereas COX-2 is the predominant source of $\mathrm{PGI}_{2}$ under resting conditions in humans $(24,34)$. The duration of the prostanoid response in both cases appears to outlast the detectable expression of COX protein evoked by LPS in circulating leucocytes.

Prior evidence for free radical generation in human endotoxemia has relied on indices that may not accurately reflect lipid peroxidation in vivo. We now report that a time-dependent increase in urinary excretion of three distinct $\mathrm{F}_{2}$-iPs coincides with the maximal con- 
stitutional response to LPS in healthy volunteers. Given the evidence that urinary iPs reflect alterations in lipid peroxidation in vivo (32), it seems likely that they will also reflect the oxidant component of inflammatory responses in humans. Although the present results pertain to experimental endotoxemia, we have previously reported that urinary $\mathrm{iPF}_{2 \alpha}-\mathrm{III}$ is increased in patients with the adult respiratory distress syndrome and that alterations in iP excretion reflect the clinical course of this disease (74).

Currently, the most commonly selected iP for analysis of oxidant stress in vivo is $\mathrm{PF}_{2 \alpha}-\mathrm{III}$. However, we have previously shown that this particular iP may also be formed by either COX isoform in vitro $(31,45)$. COX activation and oxidant stress coincide in settings such as endotoxemia $(74,75)$ and ischemia-reperfusion injury $(76,77)$ and in response to xenobiotics such as cigarettes $(78,79)$. Therefore COX-dependent formation of $\mathrm{PPF}_{2 \alpha}$-III might undermine the value of measurements of this iP as an index of lipid peroxidation in vivo. Using COX inhibitors, we have noted that this enzymatic pathway does not contribute to urinary iPF $2 \alpha$-III in healthy volunteers (30) or in cigarette smokers, a syndrome of COX-1 activation (71). We now report that pharmacologic inhibition of COX-1 or both isozymes fail to abrogate the LPS-induced increment in urinary excretion of this iP. Thus, COXdependent pathways fail to contribute detectably to urinary iPF $\mathrm{PF}_{2 \alpha}$-III, even in circumstances of marked activation of both isozymes. The striking correlation of urinary $\mathrm{iPF}_{2 \alpha}$-III with $\mathrm{iPF}_{2 \alpha}$-VI $(r=0.69 ; P<0.0001)$ also supports the notion both are formed by a common pathway, i.e., free radical catalyzed peroxidation of arachidonic acid. Similar to our observation with $\mathrm{iPF}_{2 \alpha}-\mathrm{III}$ and $\mathrm{iPF}_{2 \alpha}-\mathrm{VI}$, LPS-evoked increases in generation of 8,12-iso-iPF $2 \alpha-\mathrm{VI}$ are also unaffected by either celecoxib or ibuprofen. Thus, despite marked activation of both COX isoforms by LPS, neither appears to contribute substantially to the coincidental increment in lipid peroxidation. The prostaglandin and iP responses to LPS in humans differ in two other respects. First, although the prostanoid response was clearly related to the doses of LPS, the iP response was not. Second, the magnitude of the maximal increments in urinary prostanoids and iPs (roughly tenfold versus threefold, respectively) evoked by LPS and the duration of the responses were quite different.

In summary, controlled administration of LPS evoked an inflammatory response in humans that is accompanied by regulated expression of both COX isozymes ex vivo. These results provide molecular and pharmacologic evidence consistent with a role for both COX-1 and COX-2 in inflammation. Although COX-2 is the predominant source, $\mathrm{COX}-1$ also contributes to the marked increase in formation of PGs evoked in LPS. By contrast, neither COX isozyme is an important source of lipid peroxidation in this human model of inflammation. Thus, the efficacy of combined therapy with antioxidants and COX inhibitors might be rationally investigated in syndromes in which oxidant stress and COX activation coincide.

\section{Acknowledgments}

Supported by grants (HL 54500 and MO 1RR00040) from the National Institutes of Health (NIH) and in part by a grant from Searle/Monsanto. I.A. Mardini was supported in part by NIH/National Institute for General Medical Sciences grant T32-GM07612. G.A. FitzGerald is the Robinette Foundation Professor of Cardiovascular Medicine.

1. FitzGerald, G.A. 1992. Prostaglandins and related compounds. In Cecil textbook of medicine. 19th edition. J.B. Wyngaarden, L.H. Smith, and J.C. Bennett, editors. W.B. Saunders Co. Philadelphia, Pennsylvania, USA. 1271-1276.

2. Dubois, R.N., et al. 1998. Cyclooxygenase in biology and disease. FASEB J. 12:1063-1073.

3. Smith, W.L. 1992. Prostanoid biosynthesis and mechanisms of action. Am. J. Physiol. 263:F181-F191.

4. Funk, C.D., Funk, L.B., Kennedy, M.E., Pong, A.S., and FitzGerald, G.A. 1991. Human platelet/erythroleukemia cell prostaglandin G/H synthase: cDNA cloning, expression, and gene chromosomal assignment. FASEB J. 5:2304-2312

5. Smith, W.L., Garavito, R.M., and DeWitt, D.L. 1996. Prostaglandin endoperoxide $\mathrm{H}$ synthases (cyclooxygenases)-1 and -2. J. Biol. Chem. 271:33157-33160.

6. Jones, D.A., Carlton, D.P., McIntyre, T.M., Zimmerman, G.A., and Prescott, S.M. 1993. Molecular cloning of human prostaglandin endoperoxide synthase type II and demonstration of expression in response to cytokines. J. Biol. Chem. 268:9049-9054.

7. Kujubu, D.A., Reddy, S.T., Fletcher, B.S., and Herschman, H.R. 1993. Expression of the protein product of the prostaglandin synthase2/TIS10 gene in mitogen-stimulated Swiss 3T3 cells. J. Biol. Chem. 268:5425-5430.

8. Xie, W., Chipman, J.G., Robertson, D.L., Erikson, R.L., and Simmons, D.L. 1991. Expression of a mitogen-responsive gene encoding prostaglandin synthase is regulated by mRNA splicing. Proc. Natl. Acad. Sci. USA. 88:2692-2696.

9. Niiro, H., et al. 1997. Regulation by interleukin-10 and interleukin-4 of cyclooxygenase-2 expression in human neutrophils. Blood. 89:1621-1628.

10. Masferrer, J.L., et al. 1994. In vivo glucocorticoids regulate cyclooxygenase- 2 but not cyclooxygenase- 1 in peritoneal macrophages. J. Pharmacol. Exp. Ther. 270:1340-1344.

11. Seibert, K., et al. 1994. Pharmacological and biochemical demonstration of the role of cyclooxygenase 2 in inflammation and pain. Proc. Natl. Acad. Sci. USA. 91:12013-12017.

12. Masferrer, J.L., et al. 1994. Selective inhibition of inducible cyclooxygenase 2 in vivo is antiinflammatory and nonulcerogenic. Proc. Natl. Acad. Sci. USA. 91:3228-3232.

13. Zhang, Y., Shaffer, A., Portanova, J., Seibert, K., and Isakson, P.C. 1997. Inhibition of cyclooxygenase-2 rapidly reverses inflammatory hyperalgesia and prostaglandin $\mathrm{E}_{2}$ production. J. Pharmacol. Exp. Ther. 283:1069-1075

14. O'Sullivan, M.G., Huggins, E.M., Meade, E.A., DeWitt, D.L., and McCall, C.E. 1992. Lipopolysaccharide induces prostaglandin $\mathrm{H}$ synthase- 2 in alveolar macrophages. Biochem. Biophys. Res. Commun. 187:1123-1127.

15. Masferrer, J.L., Zweifel, B.S., Seibert, K., and Needleman, P. 1990. Selective regulation of cellular cyclooxygenase by dexamethasone and endotoxin in mice. J. Clin. Invest. 86:1375-1379.

16. Martich, G.D., Boujoukos, A.J., and Suffredini, A.F. 1993. Response of man to endotoxin. Immunobiology. 187:403-416.

17. Cassale, T.B., Ballas, Z.K., Kaliner, M.A., and Keahey, T.M. 1990. The effects of intravenous endotoxin on various host-effector molecules. $J$. Allergy Clin. Immunol. 85:45-51.

18. van Deventer, S.J., et al. 1990. Experimental endotoxemia in humans: analysis of cytokine release and coagulation, fibrinolytic, and complement pathways. Blood. 76:2520-2526.

19. Bernard, G.R., et al. 1991. Prostacyclin and thromboxane $A_{2}$ formation is increased in human sepsis syndrome. Effects of cyclooxygenase inhibition. Am. Rev. Respir. Dis. 144:1095-1101.

20. Martich, G.D., Parker, M.M., Cunnion, R.E., and Suffredini, A.F. 1992. Effects of ibuprofen and pentoxifylline on the cardiovascular response of normal humans to endotoxin. J. Appl. Physiol. 73:925-931.

21. Revhaug, A., et al. 1998. Inhibition of cyclo-oxygenase attenuates the metabolic response to endotoxin in humans. Arch. Surg. 123:162-170.

22. Bernard, G.R., et al. 1997. The effects of ibuprofen on the physiology and survival of patients with sepsis. N. Engl. J. Med. 336:912-918.

23. Catella, F., and FitzGerald, G.A. 1987. Paired analysis of urinary thromboxane $\mathrm{B}_{2}$ metabolites in humans. Thromb. Res. 47:647-656.

24. McAdam, B.F., et al. 1999. Systemic biosynthesis of prostacyclin by 
cyclooxygenase (COX)-2: the human pharmacology of a selective inhibitor of COX-2. Proc. Natl. Acad. Sci. USA. 96:272-277.

25. FitzGerald, G.A., Pedersen, A.K., and Patrono, C. 1983. Analysis of prostacyclin and thromboxane biosynthesis in cardiovascular disease. Circulation. 67:1174-1177.

26. Lawson, J.A., Rokach, J., and FitzGerald, G.A. 1999. Minireview: isoprostanes: formation, analysis and use as indices of lipid peroxidation in vivo. J. Biol. Chem. 35:24441-24444.

27. Wendelborn, D.F., Seibert, K., and Roberts, L.J., II. 1999. Isomeric prostaglandin $\mathrm{F}_{2}$ compounds arising from the prostaglandin $\mathrm{D}_{2}$ : a family of icosanoids produced in vivo in humans. Proc. Natl. Acad. Sci. USA. 85:304-308.

28. Harrison, K.A., and Murphy, R.C. 1995. Isoleukotrienes are biologically active free radical products of lipid peroxidation. J. Biol. Chem. 29:17273-17278.

29. Mallat, Z., et al. 1999. The relationship of hydroxyeicosatetraenoic acids and $\mathrm{F}_{2}$-isoprostanes to plaque instability in human carotid atherosclerosis. J. Clin. Invest. 103:421-427.

30. Praticò, D., et al. 1998. IPF $2 \alpha-\mathrm{I}$ : an index of lipid peroxidation in humans. Proc. Natl. Acad. Sci. USA. 95:3449-3454.

31. Praticò, D., Lawson, J.A., and FitzGerald, G.A. 1995. Cyclooxygenase dependent formation of 8-epi PGF P $_{2 \alpha}$ in human platelets. J. Biol. Chem. 270:9800-9808.

32. Lawson, J., et al. 1998. Identification of two major $F_{2}$ isoprostanes, 8,12iso- and 5-epi-8,12-iso-isoprostane-VI, in human urine. J. Biol. Chem. 273: 29295-29301.

33. Li, W., Lawson, J., Reilley, M., Rokach, J., and FitzGerald, G.A. 1999. Quantitative analysis of $\mathrm{F}_{2}$ isoprostanes by HPLC/tandem mass spectrometry. Proc. Natl. Acad. Sci. USA. 96:13381-13386.

34. Smith, W.L., Meade, E.A., and DeWitt, D.L. 1994. Pharmacology of prostaglandin endoperoxide synthase isozymes-1 and -2. In Plateletdependent vascular occlusion. G.A. FitzGerald, L.K. Jennings, and C. Patrono, editors. The New York Academy of Sciences. New York, New York, USA. 136-142.

35. Catella-Lawson, F., et al. 1999. Effects of specific inhibition of cyclooxygenase-2 on sodium balance, hemodynamics, and vasoactive eicosanoids. J. Pharmacol. Exp. Ther. 289:735-741.

36. Towbin, H., Staehelin, T., and Gordon, J. 1979. Electrophoretic transfer of proteins from polyacrylamide gels to nitrocellulose sheets: procedure and some applications. Proc. Natl. Acad. Sci. USA. 76:4350-4354.

37. Creminon, C., et al. 1995. Immunological studies of human constitutive cyclooxygenase (COX-1) using enzyme immunometric assay. Biochim. Biophys. Acta. 1254:333-340.

38. Creminon, C., et al. 1995. Differential measurement of constitutive $(\mathrm{COX}-1)$ and inducible (COX-2) cyclooxygenase expression in human umbilical vein endothelial cells using specific immunometric enzyme immunoassays. Biochim. Biophys. Acta. 1254:341-348.

39. Christ, W.J., et al. 1995. E5531, a pure endotoxin antagonist of high potency. Science. 268:80-83.

40. Schumann, R.R., et al. 1990. Structure and function of lipopolysaccharide binding protein. Science. 249:1429-1431.

41. Wright, S.D., Ramos, R.A., Tobias, P.S., Ulevitch, R.J., and Mathison, J.C. 1990. CD14, a receptor for complexes of lipopolysaccharide (LPS) and LPS binding protein. Science. 249:1431-1433.

42. Yang, R.B., et al. 1998. Toll-like receptor- 2 mediates lipopolysaccharideinduced cellular signalling. Nature. 395:284-288.

43. Chow, J.C., Young, D.W., Golenbock, D.T., Christ, W.J., and Gusovsky, F. 1999. Toll-like receptor-4 mediates lipopolysaccharide-induced signal transduction. J. Biol. Chem. 274:10689-10692.

44. Hempel, S.L., Monick, M.M., and Hunninghake, G.W. 1994. Lipopolysaccharide induces prostaglandin $\mathrm{H}$ synthase-2 protein and mRNA in human alveolar macrophages and blood monocytes. J. Clin. Invest. 93:391-396.

45. Praticó, D., and FitzGerald, G.A. 1996. Generation of 8-epi PGF $2 \alpha$ by human monocytes: discriminate production by reactive oxygen species and PG G/H S-2. J. Biol. Chem. 271:8919-8924.

46. van der Poll, T., van Deventer, S.J., Buller, H.R., Sturk, A., and ten Cate, J.W. 1991. Comparison of the early dynamics of systemic prostacyclin release after administration of tumor necrosis factor and endotoxin to healthy humans. J. Infect. Dis. 164:599-601.

47. Simon, L.S., et al. 1999. Anti-inflammatory and upper gastrointestinal effects of celecoxib in rheumatoid arthritis. A randomized controlled trial. JAMA. 282:1921-1928.

48. Langman, M.J., et al. 1999. Adverse upper gastrointestinal effects of rofecoxib compared with NSAIDs. JAMA. 282:1929-1933.

49. Langenbach, R., et al. 1995. Prostaglandin synthase 1 gene disruption in mice reduces arachidonic acid-induced inflammation and indomethacin-induced gastric ulceration. Cell. 83:483-492.

50. Morham, S.G., et al. 1995. Prostaglandin synthase 2 gene disruption causes severe renal pathology in the mouse. Cell. 83:473-482.

51. Patrono, C. 1994. Aspirin as an antiplatelet drug. N. Engl. J. Med. 330:1287-1294.
52. Ushikubi, F., et al. 1998. Impaired febrile response in mice lacking the prostaglandin E receptor subtype EP3. Nature. 395:281-284.

53. Murata, T., et al. 1997. Altered pain perception and inflammatory response in mice lacking prostacyclin receptor. Nature. 388:678-682.

54. FitzGerald, G.A., Friedman, L.A., Miyamori, I., O'Grady, J., and Lewis, P.J. 1979. A double blind placebo controlled crossover study of prostacyclin in man. Life Sci. 25:665-672.

55. Salat, A., et al. 1999. Endotoxin enhances in vitro platelet aggregability in whole blood. Thromb. Res. 93:145-148.

56. Masferrer, J.L., Zweifel, B.S., Seibert, K., and Needleman, P. 1990. Selective regulation of cellular cyclooxygenase by dexamethasone and endotoxin in mice. J. Clin. Invest. 86:1375-1379.

57. Habib, A., et al. 1993. Demonstration of an inducible cyclooxygenase in human endothelial cells using antibodies raised against the carboxyl-terminal region of the cyclooxygenase-2. J. Biol. Chem. 268:23448-23454.

58. Hurley, J.C. 1995. Endotoxemia: methods of detection and clinical correlates. Clin. Microbiol. Rev. 8:268-292.

59. Boujoukos, A.J., Martich, G.D., Supinski, E., and Suffredini, A.F. 1993. Compartmentalization of the acute cytokine response in humans after IV endotoxin administration. J. Appl. Physiol. 74:3027-3033.

60. Richardson, R.P., et al. 1989. Peripheral blood leukocyte kinetics following in vivo LPS administration to normal human subjects. Ann. Surg. 210:239-245.

61. O'Sullivan, M.G., Chilton, F.H., Huggins, E.M., Jr., and McCall, C.E. 1992. Lipopolysaccharide priming of alveolar macrophages for enhanced synthesis of prostanoids involves induction of a novel prostaglandin H synthase. J. Biol. Chem. 267:14547-14550.

62.Jun, S.S., Chen, Z., Pace, M.C., and Shaul, P.W. 1999. Glucocorticoids downregulate cyclooxygenase- 1 gene expression and prostacyclin synthesis in fetal pulmonary artery endothelium. Circ. Res. 84:193-200.

63. Schonbeck, U., Sukhova, G.K., Graber, P., Coulter, S., and Libby, P. 1999. Augmented expression of cyclooxygenase-2 in human atherosclerotic lesions. Am. J. Pathol. 155:1281-1291.

64. Crofford, L.J., et al. 1994. Cyclooxygenase-1 and -2 expression in rheumatoid synovial tissues. Effects of interleukin-1 beta, phorbol ester, and corticosteroids. J. Clin. Invest. 93:1095-1101.

65. Maloney, C.G., et al. 1998. Inflammatory agonists induce cyclooxygenase type 2 expression by human neutrophils. J. Immunol. 160:1402-1410.

66. Wennmalm, A., and Fitzgerald, G.A. 1988. Excretion of prostacyclin and thromboxane $\mathrm{A}_{2}$ metabolites during leg exercise in humans. Am. J. Physiol. 255:H15-H18.

67. Roy, L., Knapp, H.R., Robertson, R.M., and FitzGerald, G.A. 1985. Endogenous biosynthesis of prostacyclin during cardiac catheterization and angiography in man. Circulation. 71:434-440.

68. FitzGerald, G.A., Smith, B., Pedersen, A.K., and Brash, A.R. 1984. Increased prostacyclin biosynthesis in patients with severe atherosclerosis and platelet activation. N. Engl. J. Med. 310:1065-1068.

69. Reilly, I.A., Doran, J.B., Smith, B., and FitzGerald, G.A. 1986. Increased thromboxane biosynthesis in a human preparation of platelet activation: biochemical and functional consequences of selective inhibition of thromboxane synthase. Circulation. 73:1300-1309.

70. Fitzgerald, D.J., Roy, L., Catella, F., and FitzGerald, G.A. 1986. Platelet activation in unstable coronary disease. N. Engl. J. Med. 315:983-989.

71. Maier, J.A., Hla, T., and Maciag, T. 1990. Cyclooxygenase is an immediate-early gene induced by interleukin-1 in human endothelial cells. J. Biol. Chem. 265:10805-10808.

72. Rimarachin, J.A., et al. 1994. Regulation of cyclooxygenase-2 expression in aortic smooth muscle cells. Arterioscler. Thromb. 14:1021-1031.

73. Topper, J.N., Cai, J., Falb, D., and Gimbrone, M.A., Jr. 1996. Identification of vascular endothelial genes differentially responsive to fluid mechanical stimuli: cyclooxygenase-2, manganese superoxide dismutase, and endothelial cell nitric oxide synthase are selectively up-regulated by steady laminar shear stress. Proc. Natl. Acad. Sci. USA. 93:10417-10422.

74. Reilly, M.P., et al. 1998. Isoprostanes in the assessment of oxidant stress in vivo. In Molecular and cellular basis of inflammation. C.N. Serhan and P.A Ward, editors. Humana Press. Totowa, New Jersey, USA. 127-139.

75. Geisel, J., Cook, J.A., Ashton, S.H., Wise, W.C., and Halushka, P.V. 1994. Endotoxin tolerance is associated with decreased prostaglandin $\mathrm{H}$ synthases-1 and -2. Am. J. Physiol. 267:C1067-C1072.

76. Kerins, D.M., Roy, L., FitzGerald, G.A., and Fitzgerald, D.J. 1989. Platelet and vascular function during coronary thrombolysis with tissue-type plasminogen activator. Circulation. 80:1718-1725.

77. Delanty, N., et al. 1997. 8-epi $\mathrm{PGF}_{2 \alpha}$ generation during coronary reperfusion: a potential quantitative marker of oxidant stress in vivo. Circulation. 95:2492-2499.

78. Nowak, J., Murray, J.J., Oates, J.A., and FitzGerald, G.A. 1987. Biochemical evidence of a chronic abnormality in platelet and vascular function in apparently healthy chronic cigarette smokers. Circulation. 76:6-14.

79. Reilly, M., Delanty, N., Lawson, J.A., and FitzGerald, G.A. 1996. Modulation of oxidant stress in vivo in chronic cigarette smokers. Circulation. 94:19-25 